This item was submitted to Loughborough's Research Repository by the author.

Items in Figshare are protected by copyright, with all rights reserved, unless otherwise indicated.

\title{
Auto-appraisal of voltage rating for switched-capacitor DC-DC converters
}

PLEASE CITE THE PUBLISHED VERSION

https://doi.org/10.1049/icp.2021.0993

PUBLISHER

IET

VERSION

AM (Accepted Manuscript)

PUBLISHER STATEMENT

This paper is a postprint of a paper submitted to and accepted for publication in The 10th International Conference on Power Electronics, Machines and Drives (PEMD 2020) and is subject to Institution of Engineering and Technology Copyright. The copy of record is available at the IET Digital Library.

\section{LICENCE}

CC BY-NC-ND 4.0

\section{REPOSITORY RECORD}

Taghizadeh, H, Andrew Cross, and N Narayanan-Kuruveettil. 2021. "Auto-appraisal of Voltage Rating for Switched-capacitor DC-DC Converters". Loughborough University. https://hdl.handle.net/2134/16950271.v1. 


\title{
AUTO-APPRAISAL OF VOLTAGE RATING FOR SWITCHED-CAPACITOR DC-DC CONVERTERS
}

\author{
Hassan Taghizadeh ${ }^{1}$, Andrew Cross $^{1^{*}}$, Nakul Narayanan-Kuruveettil ${ }^{2}$ \\ ${ }^{1}$ School of Mechanical, Electrical and Manufacturing Engineering, Loughborough University, UK \\ ${ }^{2}$ Indian Institute of Science Bangalore, Bangalore, India \\ *A.Cross@lboro.ac.uk
}

Keywords: SWITCHED CAPACITOR, SYNTHESIS, OPTIMISATION, VOLTAGE RATING

\begin{abstract}
Resonant Switched Capacitor (SC) DC-DC converters are showing potential for use in medium to high-power applications. The high-cell count in these applications means that for a given voltage conversion ratio there are theoretically a large number of alternative topologies that can be synthesised. However, each of these topologies may exhibit different competing characteristics, for example total VA rating of capacitors, total VA rating of switches, number of switches and individual switch voltage rating. Obtaining an optimum topology in terms of given design constraints is therefore important requirement. This paper builds on previous work by the authors by presenting a method for the automatic calculation of the voltage ratings of the components in a SC converter. This result along with earlier work, allows for the calculation of VA ratings, which are an important metric when comparing competing circuits. In addition, a new Canonical Cell topology is presented that extends the range of SC circuits that can be analysed using this method, to cover so-called Ladder circuits. The method is validated against detailed Spice simulations and measurements from a hardware prototype.
\end{abstract}

\section{Introduction}

Switched Capacitor DC-DCs converters are suited to high voltage-conversion ratio applications, with the advantage that they do not need large, heavy inductors/transformers in the conversion process. They are therefore possible candidates for the collector platform of off-shore wind farms, where the DC voltage is stepped-up for transmission back to shore and size/weight of the DC-DC converter is of premium concern. High-power, SC converters are implemented using so-called resonant SC topologies, reduces the peak current stress on the circuit components and offers soft-switching [1-3].

The cellular nature of SC converters means there is often a large number of combinations in which the topology can be realised for a given voltage conversion ratio. The work described in this paper forms part of study that is developing a computer algorithm that automatically identifies the optimum SC topology from the combinations, based on a particular design goal [4]. For example, the minimisation of the converter size/weight for off-shore HVDC platforms where total capacitor VA is the dominant factor. The process proposed in [4] consists of first generating a candidate topology termed auto-synthesis, followed by auto-appraisal of the circuit, which includes the calculation of component current ratings, voltage ratings, VA ratings and the total number of components. These latter criteria are used as inputs to an optimisation routine, which drives the auto-synthesis routine toward the optimal topology.

Other techniques for auto-synthesis of SC circuits have been proposed in the literature but are either very complex, inefficient or restricted to converters with a low voltage conversion ratio [5-7]. The unique feature of the method proposed here and in [4] is the use of a Canonical Cell topology
- previously termed a Basic Cell in [4]. However, the initial work presented in [4], is lacking in two important areas: (i) the proposed Canonical Cell topology could not synthesise socalled Ladder topologies and (ii) the Appraisal process did not include the calculation of voltage ratings. Both these limitations are addressed in this paper. The paper first proposes a new Canonical Cell circuit, which is followed by the description of a new state-equation analysis and is used to calculate steady-state circuit node voltages. As in [4], such a state-equation based analysis lends itself to computer automation using a recursive algorithm. The results of the analysis are used in the paper to obtain the VA ratings of some well-known converters, which are validated against a detailed Spice simulation. Finally, as a demonstration of the technique a new circuit is auto-synthesised using a random assignment of cell connections, the topology is then auto-appraised and the calculation of voltage ratings of its components from the autoappraisal process are validated against measurements from a hardware prototype.

\section{General, Canonical Switched Capacitor Cell and Synthesis of SC Circuits}

The new Canonical Switched Capacitor Cell is shown in Fig. 1, where the $j$ subscript denotes the $j^{\text {th }}$ cell of an $N$ cell $\mathrm{SC}$ converter. It can be seen that the cell capacitor and switch $S_{1}$ form a sample-and-hold circuit which is controlled by a $50 \%$ duty-cycle gate-signal $g_{j}$, with the low potential terminal of the capacitor connected to a half-bridge leg consisting of switches $S_{2}$ and $S_{3}$. Note the annotation of switches $S_{2}$ and $S_{3}$ has been interchanged in this paper compared with the previous circuit presented in [4]. 

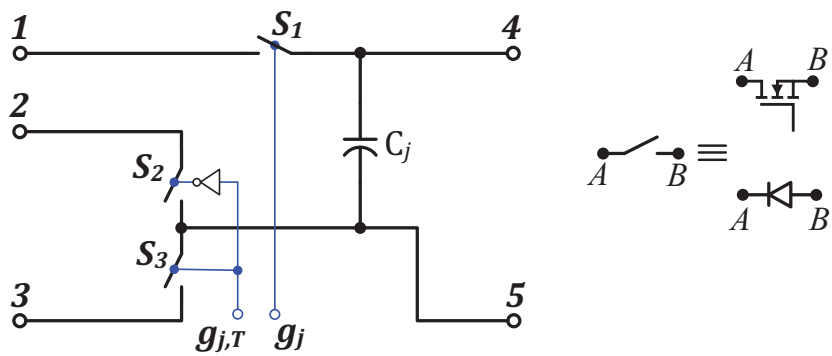

Fig. 1 Modified Canonical Switched Capacitor Cell

Unlike the cell proposed in [4], this new cell has an additional control signal $g_{j, T}$. This gives the cell extra flexibility and allows it to be used for so-called Ladder SC structures. The new control signal $g_{j, T}$ can be used as a gate signal by connecting it to $g_{j}$ or its inverse, or it can be fed with a fixed logic signal to alter the fundamental cell topology. In the latter configuration, switches $S_{2}$ and $S_{3}$ are held in permanent states and can therefore be removed and replaced by short and/or open circuits. The cell then represents a sample-and-hold function.

Only unidirectional step-up operation of the cell is considered in the analysis described here, where power flows from left-hand terminals 1-3 of the cell to the right-hand terminals 4-5. Nevertheless, the results are equal valid for stepdown and bidirectional operation. This direction of power flow is also associated with the terms "up-stream" and "downstream" cells, which are used in this paper.

A SC circuit is composed of $N$ cells, which are interconnected to form the overall converter as shown in Fig. 2. The gate signal for each cell $g_{j}$, connects to a global gate signal $g$ or its inverse $\bar{g}$. In addition, each cell can then be configured through its topology control signal $g_{j, T}$. The converter has a DC input and output voltages $V_{\text {in }}$ and $V_{o}$ respectively.

The inter-connection of the cells was defined in [4] using a connection matrix $\boldsymbol{c}$, where each element $c_{\mathrm{ij}}$ represents the cell number to which the $i^{\text {th }}$ input of the $j^{\text {th }}$ cell shown in Fig. 2, is connected,

$$
c=\left(\begin{array}{llll}
c_{11} & c_{12} & \cdots & c_{1 N} \\
c_{21} & c_{22} & \cdots & c_{2 N} \\
c_{31} & c_{32} & \cdots & c_{3 N}
\end{array}\right)
$$

The synthesis of a converter includes synthesis rule 1 [4]: input terminals 1 and 2 can only connect to terminal 4 of a single up-stream cell or the input DC voltage $V_{\text {in }}$, whereas terminal 3, can only connect to terminal 5 of a single up-stream cell or ground. This rule ensures that for step-up operation the voltage increases monotonically from cell-to-cell, which avoids redundant cell connections. A zero entry in the cell connection matrix is defined as the connection of inputs 1 and/or 2 to the supply voltage $V_{i n}$ and input 3 to ground.

For those cells where $g_{j, T}=0$ or $g_{j, T}=1$, then the resulting cell topology is such that inputs 2 or 3 no longer exist. In which case a "don't care" $\mathrm{X}$ is input to the corresponding entry of the connection matrix.

The connection of the gate signal $g_{j}$ of the individual cells is defined by gate signal vector $\boldsymbol{G}$, where the elements of $\boldsymbol{G}$ take on values of either $g$ or $\bar{g}$,

$$
\boldsymbol{G}=\left[\begin{array}{llll}
g_{1} & g_{2} & \cdots & g_{N}
\end{array}\right]
$$

The topology input signal $g_{j, T}$, for each cell is defined using the additional vector $\boldsymbol{G}_{\boldsymbol{T}}$. Each element in the vector $\boldsymbol{G}_{\boldsymbol{T}}$ will take on values of either $g_{j}$ or its inverse, Boolean $F=$ False $=0$ or Boolean $T=$ True $=1$,

$$
\boldsymbol{G}_{\boldsymbol{T}}=\left[\begin{array}{llll}
g_{1, T} & g_{2, T} & \cdots & g_{N, T}
\end{array}\right]
$$

Table 1 shows some well-known SC circuits constructed from the proposed cell structure along with their corresponding matrices $\boldsymbol{c}, \boldsymbol{G}$ and $\boldsymbol{G}_{\boldsymbol{T}}$. All these topologies are shown with an example voltage conversion ratio of $r=4$, except the Fibonacci circuit which has a ratio of $r=5$. Note: the MMSCC converter referred to in Table 1 is the Multilevel Modular SC Converter proposed in [8].

\section{Derivation of Component Voltages for a General SC Converter}

In [4], the discrete state-equations for charge transfer were derived for the Canonical Cell and used to devise a recursive algorithm to calculate the normalised, average currents flowing through each branch of the circuit. This algorithm used the cell connection matrix $\boldsymbol{c}$ and gate-signal vector $\boldsymbol{G}$ as inputs, and its outputs were the so-called charge-multipliers [4]. In a similar way, a method is now presented that allows the circuit node voltages to be calculated normalised to the converter input voltage $V_{i n}$. This then allows the voltage across each component to be determined, and when combined with the charge-multipliers gives the normalised VA ratings of each component. Whilst charge-multipliers are purely dependent on circuit topology, an equivalent parameter for voltage does not exist. This is because voltages also depend on capacitor voltage ripple, component parasitic resistance and junction voltage drops. A voltage multiplier is therefore defined here as

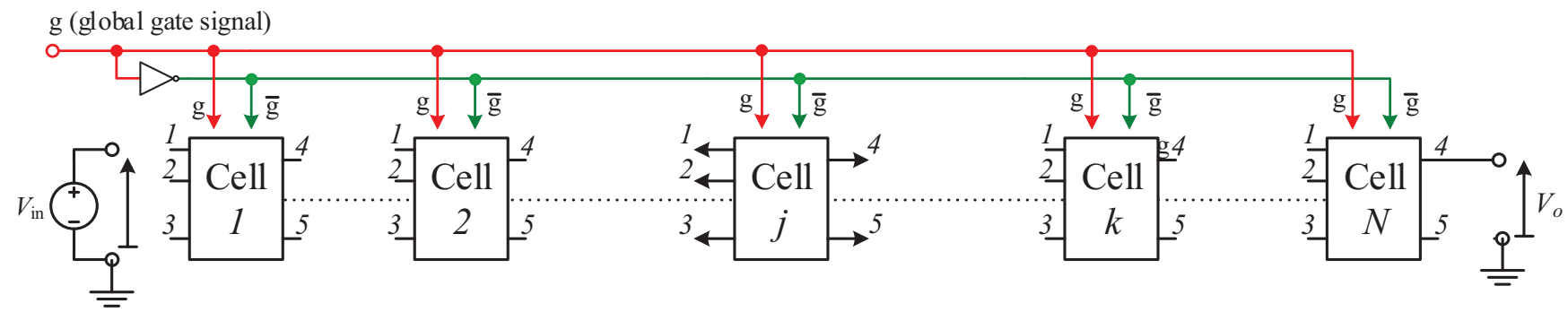

Fig. 2 General cell arrangement for a SC converter using Canonical Cells 
Table 1 SC circuits using the Canonical Cell. (a)Fibonacci, (b)Doubler, (c)Series-Parallel, (d)MMSCC, (e)Dickson, (f)Ladder

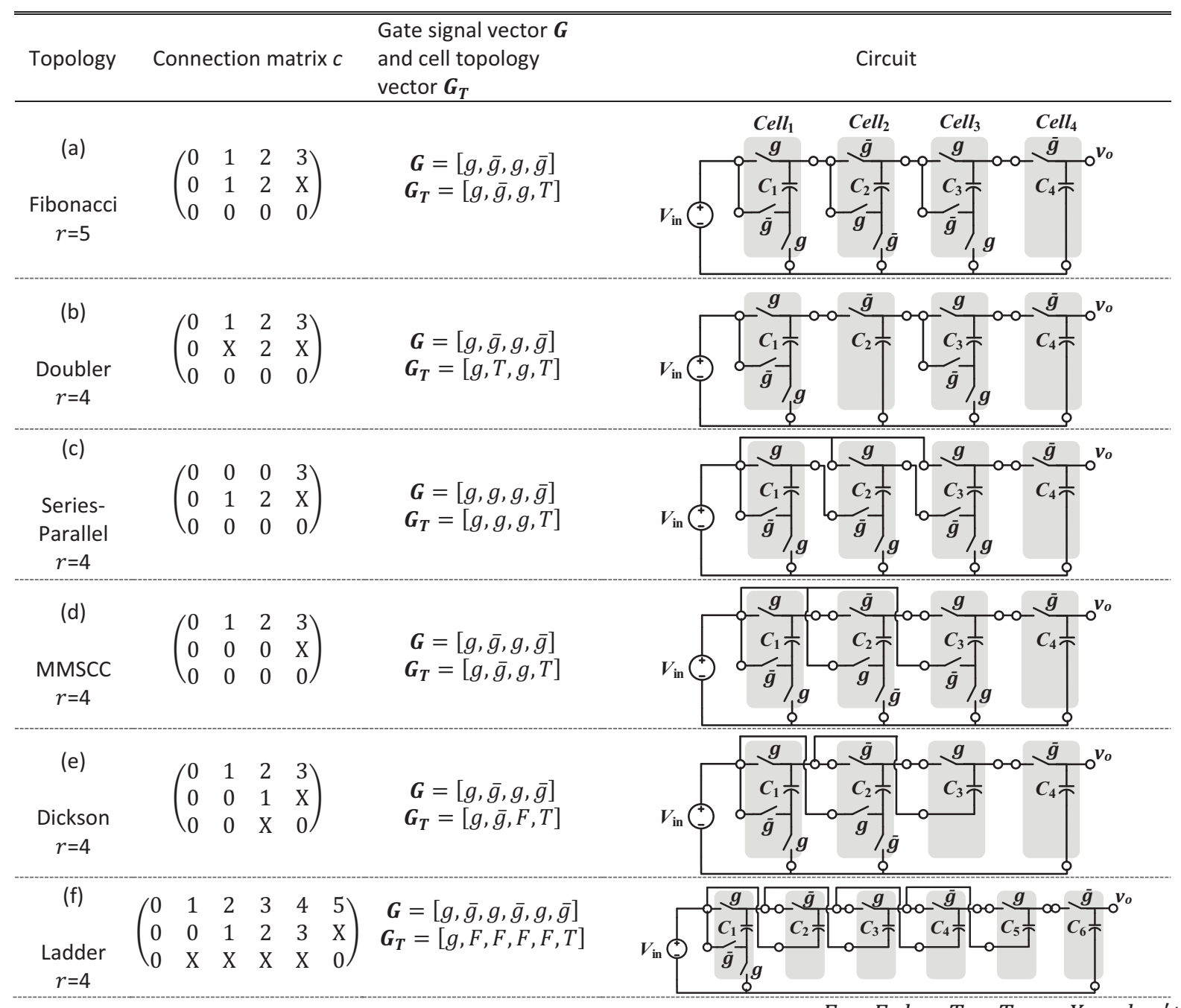

$F=$ False,$T=$ True,$X=$ don't $^{\prime}$ care

a "voltage-rating-multiplier", since for rating calculations it is assumed that these effects are negligible - this assumption also gives a more conservative value of the voltage rating.

The voltage-rating-multiplier for the components within a cell can be obtained from its terminal voltages, which in turn are obtained from the discrete state-equations for each cell. A general solution for the cell output terminal-to-ground voltages in discrete form $v_{4, j}\left[g_{j}\right]$ and $v_{5, j}\left[g_{j}\right]$, can be derived in terms of the input terminal-to-ground voltages $v_{1, j}\left[g_{j}\right]$, $v_{2, j}\left[g_{j}\right]$ and $v_{3, j}\left[g_{j}\right]$ by circuit analysis of the three possible Canonical Cell topologies:

$$
\begin{aligned}
& v_{4, j}\left[g_{j}\right] \\
& =\left\{\begin{array}{cc}
g_{j} v_{1, j}\left[g_{j}\right]+\overline{g_{j}}\left(v_{2, j}\left[g_{j}\right]+V_{C, j}\right) & g_{j, T}=g_{j} \\
v_{2, j}\left[g_{j}\right]+V_{C, j} & g_{j, T}=F \\
v_{3, j}\left[g_{j}\right]+V_{C, j} & g_{j, T}=T
\end{array}\right. \\
& v_{5, j}\left[g_{j}\right]=v_{4, j}\left[g_{j}\right]-V_{C, j}
\end{aligned}
$$

where $V_{C, j}$ is the voltage across the capacitor of the $j^{\text {th }}$ cell. Note, $g_{j, T}=\bar{g}_{j}$ is a fourth option, which will be discussed in a future paper. The capacitor voltage itself is independent of the cell state and is simply,

$$
V_{C, j}=\left\{\begin{array}{cc}
v_{1, j}[1]-v_{3, j}[1] & g_{j, T}=g_{j} \text { or } T \\
v_{1, j}[1]-v_{2, j}[1] & g_{j, T}=F
\end{array}\right.
$$

Furthermore, since the design rule stated in [4] and above dictates that the inputs to a cell are connected to the outputs of a lower potential cell, this connection being defined by the connection matrix $\boldsymbol{c}$, the input terminal voltages in (4) can be replaced by the expressions,

$$
\begin{aligned}
& v_{1, j}\left[g_{j}\right]=v_{4, c_{1 j}}\left[g_{c_{1 j}}\right] \\
& v_{2, j}\left[g_{j}\right]=v_{4, c_{2 j}}\left[g_{c_{2 j}}\right] \\
& v_{3, j}\left[g_{j}\right]=v_{5, c_{3 j}}\left[g_{c_{3 j}}\right]
\end{aligned}
$$

where the subscript $c_{\mathrm{xy}}$ takes on the value held in the corresponding elements of the connection matrix $\boldsymbol{c}$. In addition, $g_{c_{\mathrm{xy}}}$ in this equation is the gate-signal connected to the cell number also given by the value of the element $c_{\mathrm{xy}}$. Note that an element that contains a don't care entry X, corresponds to a cell that is configured through $g_{j, T}$ as a sample-and-hold cell topology. In which case this input 
terminal is floating and therefore its voltage is undefined.

Equations (4), (5) and (6) are combined to form a set of recursive equations that can be solved iteratively starting from the cells connected to the input voltage - a known boundary condition - through to the $N^{t h}$ cell. The voltage rating of a converter switch, is then obtained from its off-state voltage: switches $S_{1}$ and $S_{3}$ of cell $j$, which are open when $g_{j}=0$, and switch $S_{2}$ is open when $g_{j}=1$, so that,

$$
\begin{aligned}
& v_{S W 1 j}=v_{4, j}[0]-v_{1, j}[0] \\
& v_{S W 2 j}=v_{2, j}[1]-v_{5, j}[1] \\
& v_{S W 3 j}=v_{5, j}[0]-v_{3, j}[0]
\end{aligned}
$$

It should be noted that, expressions for $v_{S W 2 j}$ and $v_{S W 3 j}$ are only meaningful when the topology control signal cell $g_{j, T}=$ $g_{j}$, otherwise they are redundant and have been replaced by a short-circuit or open-circuit. The cell capacitor voltage rating is given directly from equation (5). Since the word "rating" in the term voltage-rating multiplier implies positive values, the polarity in (7) has been chosen to give the drain-source voltage for the MOSFETs shown in Fig. 1.

An algorithm to calculate cell terminal components voltages has been implemented in MATLAB and the code summarised by the flowchart shown in Fig. 3.

The inputs to the algorithm are the predefined connection matrix $\boldsymbol{c}$, gate control signal $\boldsymbol{G}$ and cell topology control signal

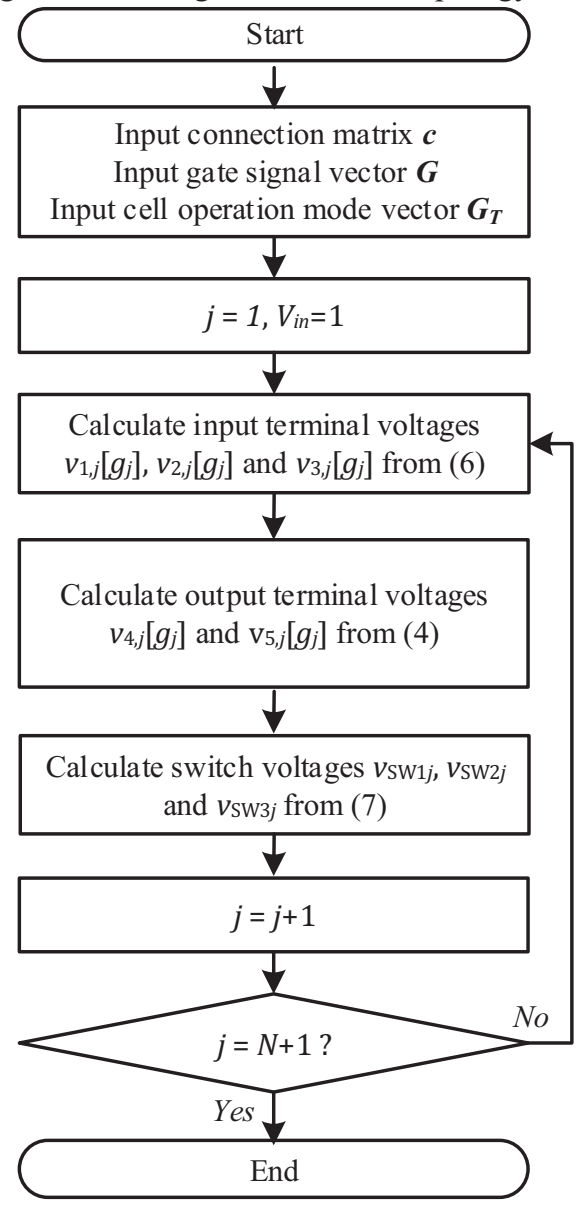

Fig. 3 Flowchart for the calculation of normalised cell terminal and component voltages. vector $\boldsymbol{G}_{\boldsymbol{T}}$. Equations (4)-(7) are coded in the algorithm as normalised values by setting the input voltage $V_{\text {in }}$ equal to 1 . In this way, the output of the algorithm directly gives the normalised voltage-rating-multipliers for the circuit. These multipliers along with the charge-multipliers calculated from the companion algorithm presented in [4], are then used for automatic computation of the normalised current, voltage and VA ratings of all switches and capacitors, and hence can be uses as a method of auto-appraisal of a candidate topology.

\section{Determination and validation of voltage-rating multipliers for well-known SC circuits}

This section presents the results of applying the proposed MATLAB-based auto-appraisal method to a number of wellknown SC circuits. In some cases, an analytic expression can be derived for the voltage-rating multipliers - similar to the example given in [4]. However, for brevity only the results of the MATLAB coded algorithm are shown here. These results have been validated against a detailed Spice simulation which are also shown for comparison. The Spice simulation included switch on-state resistances of $20 \mathrm{~m} \Omega$ and switch $S_{1}$ also included a junction voltage of $0.7 \mathrm{~V}$. This represents a unidirectional step-up converter, with cell switches $S_{2}$ and $S_{3}$ being implemented as MOSFETs and $S_{1}$ as a diode. The cell capacitor was $400 \mu \mathrm{F}$ with an ESR of $10 \mathrm{~m} \Omega$ and the switching frequency of the converter was set to $100 \mathrm{kHz}$ with $400 \mathrm{~ns}$ dead-time between the 2 switching phases. The input DC voltage was $100 \mathrm{~V}$ and the output was loaded with a $100 \Omega$ resistor when calculating charge-multipliers $\boldsymbol{a}$. These values represent typical parameters for a $1.5 \mathrm{~kW}$ residential PV converter application. The voltage-rating-multipliers $\boldsymbol{b}$ are calculated from the Spice simulation at no-load in order to minimise the effect of capacitor voltage ripple and component parasitic resistance discussed previously.

The results from executing the MATLAB algorithm and Spice simulation are shown in Table 2. The voltage-ratingmultipliers for the switches and the capacitors are given by the integer vector $\boldsymbol{b}$, and the charge-multipliers by vector $\boldsymbol{a}$, the latter corresponding to $a_{S W 1-3}$ and $a_{C}$ in [4]. The dot-product of the magnitudes of $\boldsymbol{a}$ and $\boldsymbol{b}$ when divided by the voltage conversion ratio $r$ gives the total VA switch or capacitor rating normalised to the total VA rating of the converter, $|\boldsymbol{a}| \cdot|\boldsymbol{b}| / r$. The total VA ratings for the MATLAB and Spice calculations are shown in the last two columns of the table and headed $V A_{S W}$ and $V A_{C A P}$, where it can be seen that there is very close agreement between the two methods. Small differences in the total VA rating calculations - less than $2 \%$ - are due to the diode on-state voltages used in the Spice simulation. Note that the sign of the switch charge multipliers shown in Table 2 of [4] were incorrect for $a_{S W 1}$ and $a_{S W 2}$.

\section{Validation Against a Hardware Prototype}

The connection matrix $\boldsymbol{c}$ along with the gate signal vectors $\boldsymbol{G}$ and $\boldsymbol{G}_{\boldsymbol{T}}$ were filled with random positive Integers/Booleans in order to generate a new 4-cell SC topology. This circuit was 
Table 2 Charge/voltage-rating multipliers \& total normalised VA ratings of switches \& capacitors for well-known SC converters calculated using MATLAB algorithm

\begin{tabular}{|c|c|c|c|c|c|c|c|c|c|}
\hline \multirow{2}{*}{ Topology } & \multirow{2}{*}{\multicolumn{2}{|c|}{ Switch 1}} & \multirow[b]{2}{*}{ Switch 2} & \multirow[b]{2}{*}{ Switch 3} & \multirow[b]{2}{*}{ Capacitor } & \multicolumn{2}{|c|}{ Matlab algorithm } & \multicolumn{2}{|c|}{ Spice simulation } \\
\hline & & & & & & $V A_{S W}$ & $V A_{C A P}$ & $V A_{S W}$ & $V A_{C A P}$ \\
\hline \multirow{2}{*}{$\begin{array}{c}\text { Fibonacci } \\
r=5\end{array}$} & $a$ & $-[3,2,1,1]$ & {$[2,1,1, X]$} & {$[2,1,1, X]$} & {$[2,1,1,0.5]$} & \multirow{2}{*}{4.80} & \multirow{2}{*}{1.90} & \multirow{2}{*}{4.77} & \multirow{2}{*}{1.88} \\
\hline & $b$ & {$[1,2,3,2]$} & {$[1,2,3, \mathrm{X}]$} & {$[1,1,2, \mathrm{X}]$} & {$[1,2,3,5]$} & & & & \\
\hline \multirow{2}{*}{$\begin{array}{c}\text { Doubler } \\
r=4\end{array}$} & $a$ & $-[2,2,1,1]$ & {$[2, \mathrm{X}, 1, \mathrm{X}]$} & {$[2, X, 1, X]$} & {$[2,1,1,0.5]$} & \multirow{2}{*}{4.00} & \multirow{2}{*}{2.00} & \multirow{2}{*}{3.98} & \multirow{2}{*}{1.98} \\
\hline & $b$ & {$[1,1,2,2]$,} & {$[1, X, 2, X]$} & {$[1, X, 2, X]$} & {$[1,2,2,4]$} & & & & \\
\hline \multirow{2}{*}{$\begin{array}{c}\text { Ser.-Par. } \\
r=4\end{array}$} & $a$ & $-[1,1,1,1]$ & {$[1,1,1, \mathrm{X}]$} & {$[1,1,1, X]$} & {$[1,1,1,0.5]$} & \multirow{2}{*}{4.50} & \multirow{2}{*}{1.25} & \multirow{2}{*}{4.48} & \multirow{2}{*}{1.24} \\
\hline & $b$ & {$[1,2,3,3]$} & {$[1,1,1, \mathrm{X}]$} & {$[1,2,3, \mathrm{X}]$} & {$[1,1,1,4]$} & & & & \\
\hline \multirow{2}{*}{$\begin{array}{c}\text { MMSCC } \\
r=4\end{array}$} & $a$ & $-[1,1,1,1]$ & {$[1,1,1, \mathrm{X}]$} & {$[1,1,1, \mathrm{X}]$} & {$[1,1,1,0.5]$} & \multirow{2}{*}{3.00} & \multirow{2}{*}{2.00} & \multirow{2}{*}{2.99} & \multirow{2}{*}{1.99} \\
\hline & $b$ & {$[1,2,2,1]$} & {$[1,1,1, \mathrm{X}]$} & {$[1,1,1, X]$} & {$[1,2,3,4]$} & & & & \\
\hline \multirow{2}{*}{$\begin{array}{c}\text { Dickson } \\
r=4\end{array}$} & $a$ & $-[1,1,1,1]$ & {$[2,1, X, X]$} & {$[2,1, X, X]$} & {$[2,1,1,0.5]$} & \multirow{2}{*}{3.00} & \multirow{2}{*}{2.00} & \multirow{2}{*}{2.99} & \multirow{2}{*}{1.99} \\
\hline & $b$ & {$[1,2,2,1]$} & {$[1,1, X, X]$} & {$[1,1, \mathrm{X}, \mathrm{X}]$} & {$[1,2,2,4]$} & & & & \\
\hline \multirow{2}{*}{$\begin{array}{c}\text { Ladder } \\
r=4\end{array}$} & $a$ & $-[1,1,1,1,1,1]$ & {$[3, X, \ldots X]$} & {$[3, X, \ldots X]$} & {$[3,2,2,1,1,0.5]$} & \multirow{2}{*}{3.00} & \multirow{2}{*}{2.75} & \multirow{2}{*}{2.99} & \multirow{2}{*}{2.72} \\
\hline & $b$ & {$[1,1,1,1,1,1]$} & {$[1, \mathrm{X}, \ldots \mathrm{X}]$} & {$[1, \mathrm{X}, \ldots \mathrm{X}]$} & {$[1,1,1,1,1,4]$} & & & & \\
\hline
\end{tabular}

then implemented in hardware and measurements were used as a final validation of the proposed voltage-rating multiplier calculation method. Details of the generated circuit along with calculations of its charge and voltage-rating multipliers are shown in Table 3.

The hardware protype is shown in Fig. 4. each cell consists of 3 Infineon IPP $600 \mathrm{~N} 25 \mathrm{~N} 3,250 \mathrm{~V}, 25 \mathrm{~A}, 50 \mathrm{~m} \Omega$ MOSFETs and isolated gate drives, along with a $1500 \mu \mathrm{F}$ electrolytic cell capacitor mounted underneath each PCB board.

The global clock signal, including dead-time was generated by an off-board FPGA. The MOSFETs in each cell could be configured as a discrete diode by disabling the MOSFET gatedrive and utilising its body diode. For the following tests, the converter was operated with a switching frequency of $10 \mathrm{kHz}$, a DC input voltage of $20.0 \mathrm{~V}$ and nominal output of $80.0 \mathrm{~V}$

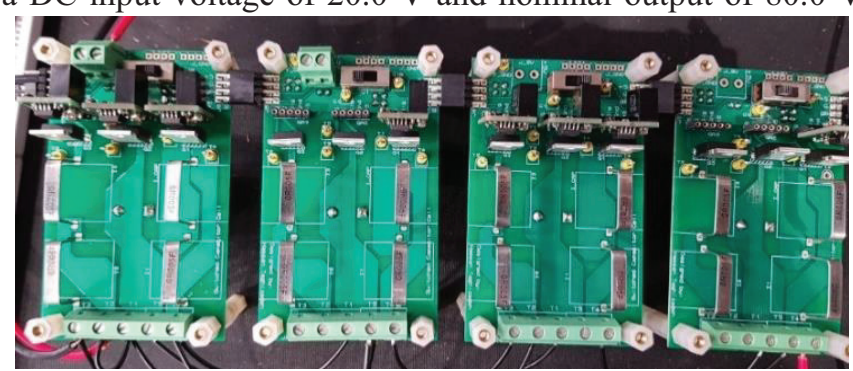

Fig. 4. Experimental prototype for the 4-Cell Switched Capacitor DC-DC converter and a load of up to $90 \mathrm{~W}$.

Initial measurements of the steady-state switch and capacitor voltages were taken at no-load and normalised against a measurement of the converter input voltage to calculate voltage-rating-multipliers. However, it was noticed that the error in these results were higher than expected. Further investigation showed that with switch $S_{1}$ implemented as a diode, energy within the parasitic circuit inductance became trapped in the cell capacitor during a switching transient, leading to a higher than calculated steady-state voltage. When $S_{1}$ was implemented as a MOSFET, this energy can recirculate back to the supply or a previous cell capacitor, where it rapidly dissipates within the circuit parasitic resistance. This effect is highlighted by measuring the outputvoltage regulation curves for $S_{1}$ configured as a diode and the MOSFET case - these results are shown in Fig. 5.

The regulation curve for the MOSFET case shows an approximate straight-line characteristic as expected, whereas the diode case shows a steep-rise in voltage as no-load is approached due to the aforementioned effect of trapped inductive energy. However, this becomes negligible with a load current of approximately $0.1 \mathrm{~A}$ and above. This value was therefore used as a pseudo-no-load current for the diode case.

Table 3 Connection and gate matrices for a randomly synthesised topology, including calculation of charge/voltage-rating multipliers and total normalised, total VA ratings of switches and capacitors using the MATLAB algorithm

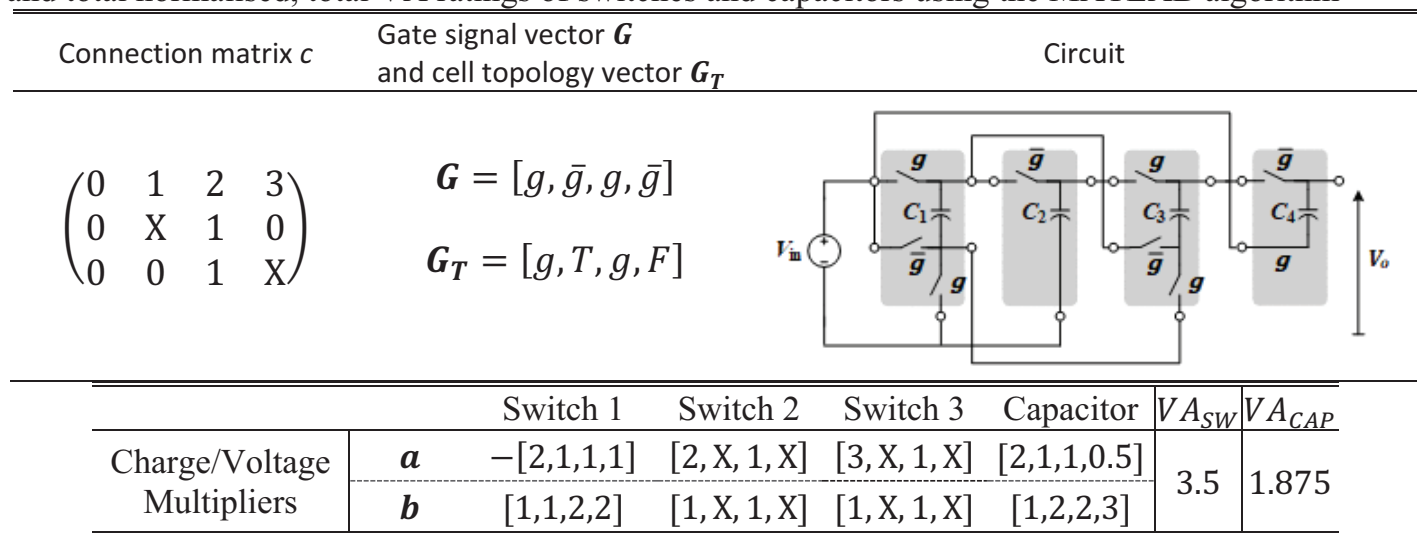


Table 4 Experimental voltage multiplier values for the arbitrary topology with both switch and diode conducting cases

\begin{tabular}{c|cccc}
\hline \hline Component & Switch 1 & Switch 2 & Switch 3 & Capacitor \\
\hline $\begin{array}{c}\text { Voltage } \\
\text { Multiplier } \boldsymbol{b}\end{array}$ & {$[0.938,0.938,1.913,1.913]$} & {$[0.963, \mathrm{X}, 0.938, \mathrm{X}]$} & {$[0.963, \mathrm{X}, 0.963, \mathrm{X}]$} & {$[0.969,1.941,1.906,2.840]$} \\
\hline
\end{tabular}

Note, the difference between the two curves in the linear region is due to the cumulative effect of the on-state junction voltage of the $S_{1}$ diodes in each cell and has a value of approximately $3.1 \mathrm{~V}$.

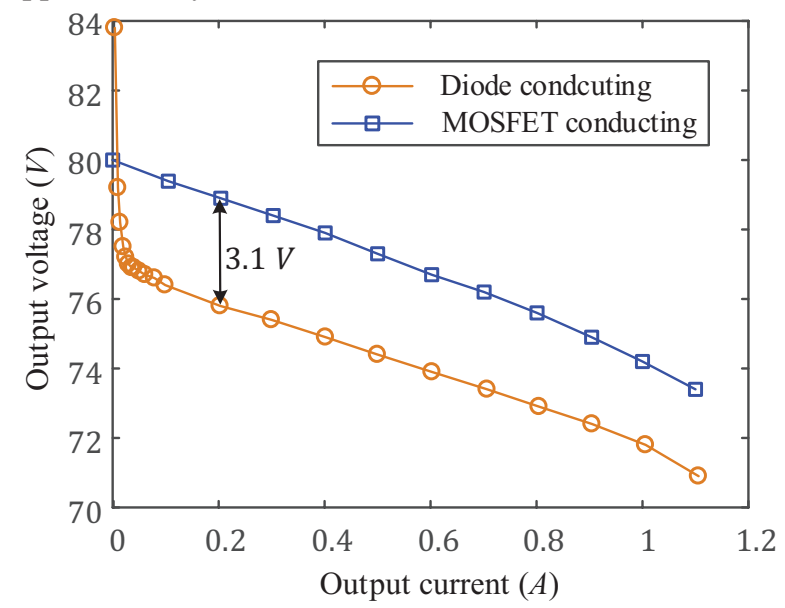

Fig. 5. Comparison of experimental voltage regulation curves for $S_{1}$ conducting as a Diode (yellow circled curve) and MOSFET (blue squared curve)

The measured voltages from the prototype at the $0.1 \mathrm{~A}$ load, which have been normalised to the converter input voltage are shown in Table 4 . The measured voltage-rating multipliers show close agreement with the $\boldsymbol{b}$ values calculated by the MATLAB algorithm shown in Table 3, the largest difference being $6.0 \%$ in the value of the first multiplier 0.938 . These differences are due to the diode on-state voltages, which have been neglected in the MATLAB algorithm.

\section{Conclusions}

This paper has proposed a method for calculating the voltage ratings of switches and capacitors in a SC converter, which when combined with the charge-multipliers can be used to calculate the VA ratings of individual components as well as a total VA rating for the converter. These voltage-rating multipliers were obtained by solving the discrete state-equations for the converter, which was then developed into a recursive algorithm to allow the automatic calculation of numerical values using MATLAB code. The method was validated against detailed Spice simulations, as well as a comparison against a hardware prototype.

A number of well-known SC converters along with the newly randomly devised circuit for the hardware prototype were appraised using VA rating as the main figure of merit.

The development of algorithms for the automatic calculation of charge-multipliers and voltage-rating multipliers presented in this and previous work opens up the opportunity for the creation of a software tool that is capable of synthesising an optimum SC circuit for a given set of design requirements.

\section{Acknowledgements}

The authors would like to express their gratitude to GE Grid Solutions, Stafford, UK and Innovate UK for funding this work through a Knowledge Transfer Partnership (KTP) as well as Carl Barker and Robert Whitehouse of GE Grid Solutions, for their technical assistance throughout the KTP project.

\section{References}

[1] H. Taghizadeh, A. M. Cross, R. Whitehouse, and C. Barker, "Switched capacitor DC-DC converters for HVDC applications," in 11th IET International Conference on $A C$ and DC Power Transmission, 10-12 Feb 2015, pp. 1-9.

[2] W. Chen, A. Huang, C. Li, and G. Wang, "A high efficiency high power step-up resonant switchedcapacitor converter for offshore wind energy systems," in 2012 IEEE Energy Conversion Congress and Exposition (ECCE), 15-20 Sept. 2012 2012, pp. 235-239, doi: 10.1109/ECCE.2012.6342818.

[3] P. Wen, X. Yang, C. Yan, T. Q. Zheng, and S. Igarashi, "Analysis of Hybrid SiC IGBT Based Resonant Switched Capacitor Converter with Circuit Parasitics Consideration," in 2019 IEEE Energy Conversion Congress and Exposition (ECCE), 29 Sept.-3 Oct. 2019 2019, pp. 4359-4365.

[4] H. Taghizadeh, A. M. Cross, R. Whitehouse, and C. Barker, "An Algorithm for Automatically Calculating Component Current Ratings in Switched-Capacitor DCDC Converters," IEEE Access, vol. 6, pp. 15702-15712, 2018.

[5] M. S. Makowski, "Realizability conditions and bounds on synthesis of switched-capacitor DC-DC voltage multiplier circuits," IEEE Transactions on Circuits and Systems I: Fundamental Theory and Applications, vol. 44, no. 8, pp. 684-691, 1997.

[6] F. Su and W. Ki, "Design Strategy for Step-Up Charge Pumps With Variable Integer Conversion Ratios," IEEE Transactions on Circuits and Systems II: Express Briefs, vol. 54, no. 5, pp. 417-421, 2007.

[7] R. Karadi, "Synthesis of switched-capacitor power converters: An iterative algorithm," in 2015 IEEE 16th Workshop on Control and Modeling for Power Electronics (COMPEL), 12-15 July 2015 2015, pp. 1-4.

[8] F. H. Khan and L. M. Tolbert, "A Multilevel Modular Capacitor-Clamped DC-DC Converter," IEEE Transactions on Industry Applications, vol. 43, no. 6, pp. 1628-1638, 2007. 